\title{
THE EARLY OUTBURST SPECTRA OF NOVA V1500 CYGNI
}

\author{
M. Mikołajewski and J. Mikołajewska \\ Institute of Astronomy,Nicolaus Copernicus University, \\ PL-87100 Torun, Poland
}

Nova cygni 1975 (V1500 Cyg) was outstanding in several respects. It was the most luminous galactic nova ever observed, had the largest outburst amplitude, and had the fastest evolution with time.

The spectra, with dispersion $28 \mathrm{~A} / \mathrm{mm}$ and in spectral range $\lambda \lambda 3560-5055 \mathrm{~A}$, were obtained with the CCS spectrograph on $90 \mathrm{~cm}$ reflector at Torun Observatory by the research group supervised by prof. A. Woszczyk. The ccs spectrograph was equipped with the 3.5 arcsec aperture Richardson's image slicer and stars with known spectral energy distributions were observed on each night, so we were able to derive absolute energy distributions for the $v 1500$ Cyg spectra. The calibration of the energy scale was performed by comparing synthetic B magnitudes calculated from our spectra with numerous published $B$ photometry of the nova. The first spectra were obtained about $3^{\text {h }}$ later than spectra reported by Boyarchuk (1977) and about $6^{\text {h }}$ earlier than spectra discussed by Duerbeck and Wolf (1977); thus they are among the earliest spectra of the nova available. Simultaneously, the spectra correspond to the earliest state of spectral evolution of a nova ouburst yet observed. The pre-maximum spectral evolution of Nova v1500 cyg is extremely fast. The spectra (Figures 1 and 2) secured on JD 2442654 are characterized by a very strong continuum and broad blueshifted absorption lines of Oll, NII and Cll of unusual strengths, as well as the Balmer series and Hel. The Balmer absorption lines are very weak on the first set of spectra and then grow stronger with time. The energy distribution of the first spectrum (JD 2442654.41) roughiy resembles that of a $B 2-B 3$ supergiant;

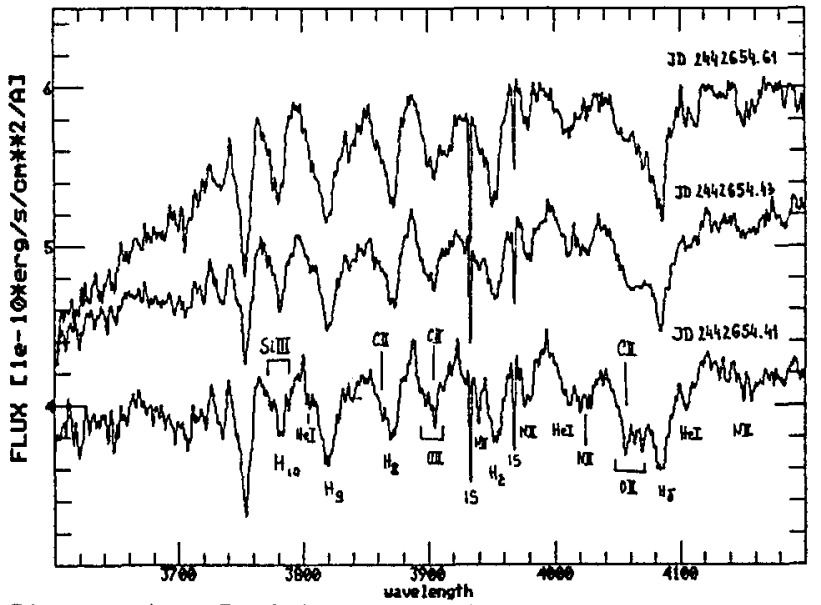

Figure 1. Evolution of the pre-maximum absorption spectrum. The flux scale of the first spectrum (bottom) has been displaced by $-10^{-10} \mathrm{erg} \mathrm{cm}^{-2} \mathrm{~s}^{-1} \mathrm{~A}^{-1}$ for better presentation. about 5 hours later (JD 2442654.61) the continuum can be compared with that of B8-B9 supergiant, although some excess flux in the UV region is present. very large equivalent widths of the Oll, NII and Cll absorption lines in comparison to those observed in normal supergiants suggest gross overabundances of carbon, nitrogen and oxygen relative to cosmic values during the early phase of the nova outburst. The radial expansion velocity of the nova envelope derived from the $H I$ Balmer absorption lines grew from about $-1250 \mathrm{~km} / \mathrm{s}$ to about $-1350 \mathrm{~km} / \mathrm{s}$ during the first 


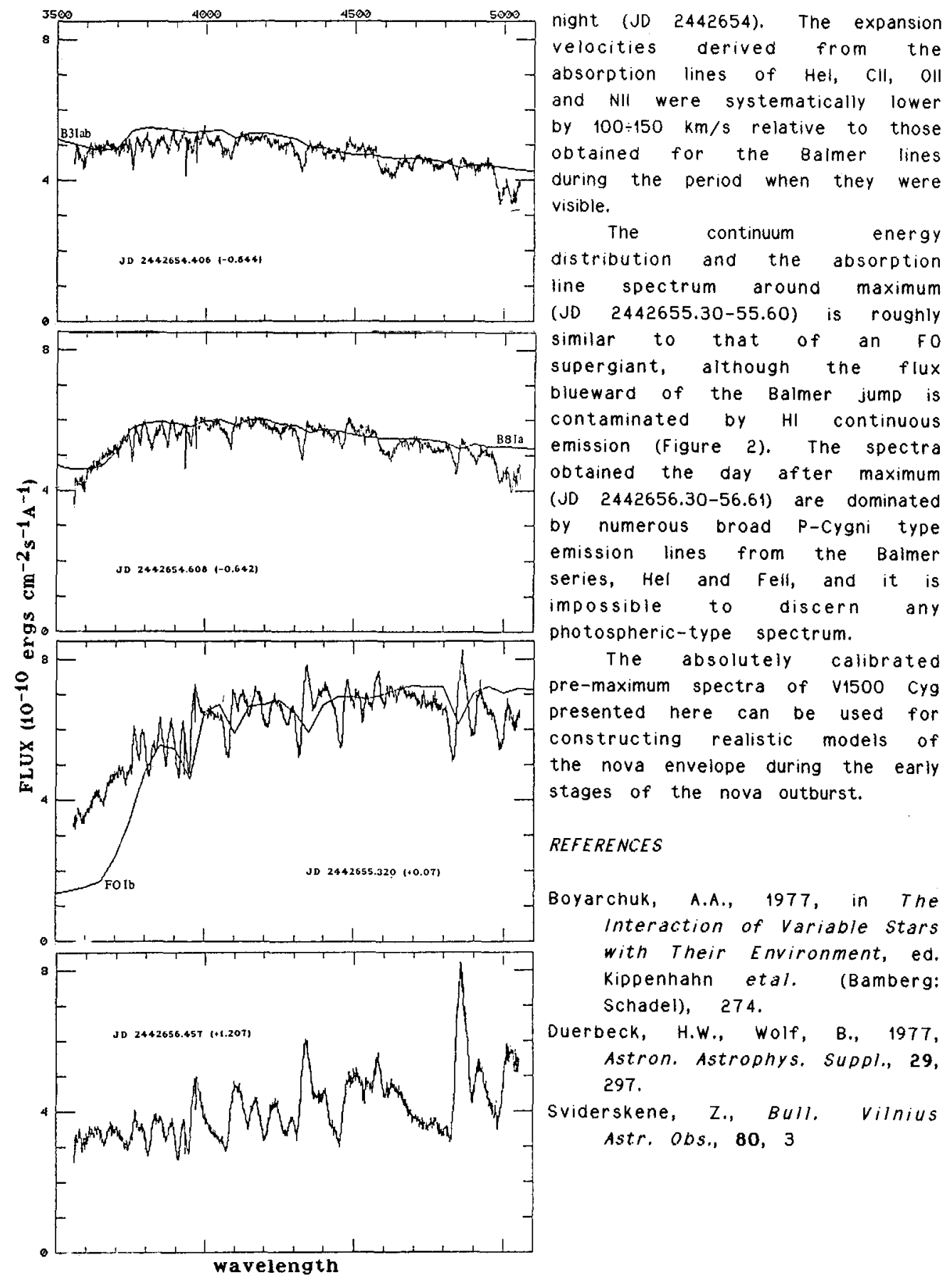

Figure 2. Absolutely calibrated spectra taken around maximum - in brackets phases (in days) relative the maximum of $B$ magnitude. Energy distributions in the supergiants spectra are from sviderskene (1988) with $E_{B-V}=0.5$. 